\title{
Gas collisions and pressure quenching of the photoluminescence of silicon nanopowder grown by plasma-enhanced chemical vapor deposition
}

\author{
P. Roura ${ }^{a)}$ and J. Costa \\ GRM, Dept. d'Enginyeria Industrial, Escola Politècnica Superior, Universitat de Girona, E17071-Girona, \\ Catalonia, Spain \\ J. R. Morante and E. Bertran ${ }^{\text {b) }}$ \\ Dept. de Física Aplicada i Electrònica, Facultat de Física, Universitat de Barcelona, E08028-Barcelona, \\ Catalonia, Spain
}

(Received 24 September 1996; accepted for publication 16 December 1996)

\begin{abstract}
The quenching of the photoluminescence of Si nanopowder grown by plasma-enhanced chemical vapor deposition due to pressure was measured for various gases $\left(\mathrm{H}_{2}, \mathrm{O}_{2}, \mathrm{~N}_{2}, \mathrm{He}, \mathrm{Ne}\right.$, Ar, and $\left.\mathrm{Kr}\right)$ and at different temperatures. The characteristic pressure, $P_{0}$, of the general dependence $I(P)$ $=I_{0} \exp \left(-P / P_{0}\right)$ is gas and temperature dependent. However, when the number of gas collisions is taken as the variable instead of pressure, then the quenching is the same within a gas family (monoor diatomic) and it is temperature independent. So it is concluded that the effect depends on the number of gas collisions irrespective of the nature of the gas or its temperature. (C) 1997 American Institute of Physics. [S0021-8979(97)01207-3]
\end{abstract}

\section{INTRODUCTION}

The luminescence of porous silicon was, at the time of its discovery, ${ }^{1}$ an unexpected phenomenon for an indirectgap semiconductor, which $\mathrm{Si}$ is. It was the beginning of a number of studies devoted to improving its radiative efficiency to make it useful for optoelectronics. In addition, several theories attempted to explain its microscopic origin by invoking a quantum-confinement effect $^{1-3}$ or the special structure of the surface. ${ }^{4,5}$ But now, other nanostructured materials based on silicon, with similar luminescent properties ${ }^{6,7}$ have been obtained, which broadens the field considerably. Among them, Si nanopowder obtained by plasma-enhanced chemical vapor deposition (PECVD) $)^{8,9}$ constitutes a special case. Its photoluminescence (PL) is excited via a multiphoton-multistep process, ${ }^{10}$ which explains its supralinear dependence on the excitation power, $\phi$,

$$
I_{\mathrm{PL}} \propto \Phi^{r}
$$

with $\mathbf{r}$ as high as 7 .

A collateral property of porous silicon luminescence is its dependence on the surrounding gas pressure. This effect is strongly dependent on the gas species. ${ }^{11}$ Polar molecules adsorbed on the surface efficiently quench the emission following an exponential law, whereas nonpolar gases have only a minor effect. Partial pressures of methanol of about 100 mbar have a pronounced effect on the PL and on the electrical conductivity of porous silicon, which could be the basis for its use in a chemical sensor. ${ }^{12}$ High hydrostatic pressures (GPa) have been applied in several studies to test theories about the microscopic origin of the luminescence. ${ }^{13}$ In the case of Si nanopowder the quenching of PL due to gas pressure is so great that it cannot be measured at atmospheric pressure. Its dependence is exponential, ${ }^{9}$

\footnotetext{
a)Electronic mail: pererg@ei.udg.es

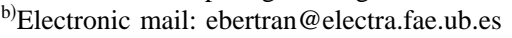

$$
I_{\mathrm{PL}}=I_{0} e^{-P / P_{0}},
$$

with $P_{0}$ of several pascals. However, in contrast to porous silicon, this effect has been observed in the presence of inert gas species and, as $P_{0}$ depends on the gas, it cannot be a hydrostatic effect. Several studies have attempted to describe this effect in Si nanopowder within the framework of the multistep-multiphoton excitation process. ${ }^{14,15}$ However, very little has been reported about the nature of the interaction between the gas and the material. In this article it is established that the effect depends on the number of gas collisions irrespective of the nature or temperature of the gas.

\section{EXPERIMENT}

The PL measurements were performed with the sample inside a closed-circuit $\mathrm{He}$ cryostat in which the temperature can be controlled from 14 to $300 \mathrm{~K}$. The luminescence was excited with the $488 \mathrm{~nm}$ line of an argon laser at about 10 $\mathrm{mW} / \mathrm{mm}^{2}$, analyzed with a $0.6 \mathrm{~m}$ monochromator, and detected with a GaAs photomultiplier. The PL emission was analyzed at a fixed wavelength $(0.8 \mu \mathrm{m})$. The gas was introduced into the cryostat through a microleak and its pressure was measured with an absolute capacitive manometer.

It was difficult to measure the pressure and the temperature of the gas around the sample at low temperatures because the temperature gradients together with the low pressure $\left(\begin{array}{lll}\sim 10 & \mathrm{~Pa}\end{array}\right)$ create nonequilibrium conditions. To overcome this difficulty the sample was placed in a box, the walls of which were held at the cold finger temperature (Fig. 1). The front of the box was transparent in order to allow PL measurements. Small holes allowed the gas to flow through the walls. It was then assumed that the gas around the sample was at the same temperature as the cold finger, which was measured with a $\mathrm{Si}$ diode. It was further assumed that the pressure of the gas around the sample was the same as the pressure at the manometer providing two conditions were 


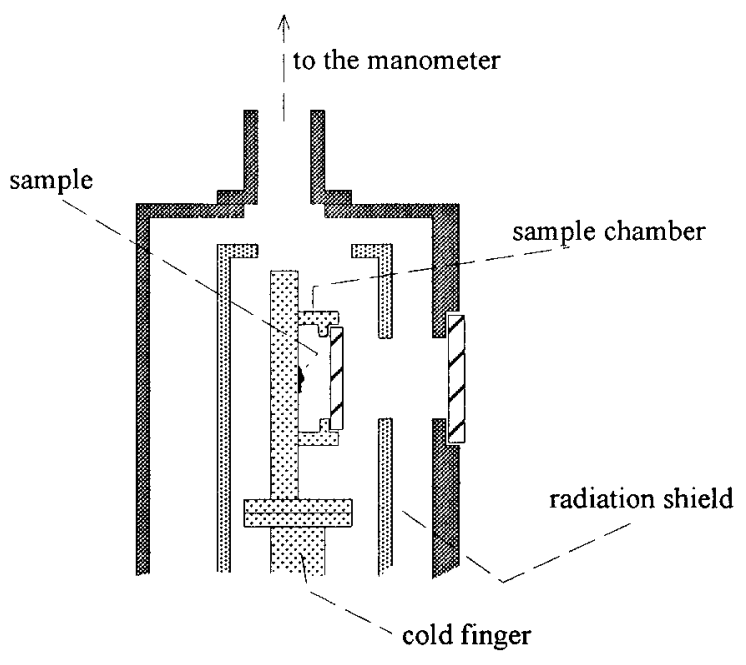

FIG. 1. A schematic view of the cryostat. The chamber around the sample ensures that the gas inside it is at equilibrium conditions. Its temperature is that of the cold finger. The manometer is placed on top to minimize the convective currents.

satisfied: (1) there is no convection and (2) the mean free path of the molecules is much smaller than the distance between the sample and the manometer. The convective currents were minimized by placing the manometer $(300 \mathrm{~K})$ on top of the cryostat. On the other hand, at typical conditions ( $3 \mathrm{~Pa}$ and $200 \mathrm{~K})$, the mean free path is small enough ${ }^{16}(\sim 2$ $\mathrm{mm})$ to satisfy condition (2).

The nominal temperature of the measurements reported in this work are assumed to correspond to the temperature of the gas surrounding the sample. Due to the very low thermal conductivity of the material, laser excitation can induce great local heating on the sample which, at present, we have not quantified. This may be the origin of the transverse optical (TO)-peak shift to lower energies in the Raman spectra of $\mathrm{Si}$ nanopowder ${ }^{17}$ rather than the residual strain as suggested by other authors. ${ }^{18}$

\section{RESULTS AND DISCUSSION}

The pressure dependence of the PL was measured with a series of gases $\left(\mathrm{H}_{2}, \mathrm{~N}_{2}, \mathrm{O}_{2}, \mathrm{He}, \mathrm{Ne}, \mathrm{Ar}\right.$, and $\left.\mathrm{Kr}\right)$ and at different temperatures. The dependence of the photoluminescence on gas pressure was found to be exponential for all the gases assayed (Fig. 2). Moreover, quenching was faster for lighter gases. This means that the characteristic pressure in Eq. (2), $P_{0}$, increases with the molecular mass. Although the quenching at different points on the same sample varied by up to $300 \%$ for a given gas, the relative values within the series of gases did not vary by more than $30 \%$. Consequently, the measurements reported in Fig. 2 have to be taken as typical results.

$P_{0}$ for He increased with temperature (Fig. 3). A similar evolution was observed for all the gases tested. The dependence of $P_{0}$ on gas and temperature is analyzed below.

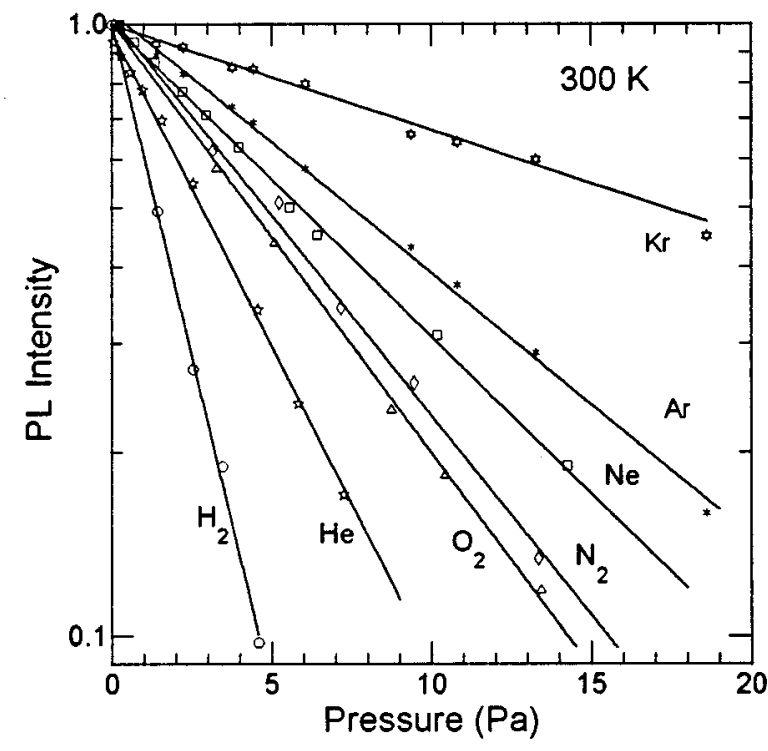

FIG. 2. Quenching of the PL intensity with different gases at room temperature.

\section{A. The pressure effect in terms of several microscopic gas variables}

The characteristic pressure, $P_{0}$, is gas dependent and varies with temperature (Fig. 3). This means that the effect of the gas on the luminescence is not hydrostatic. Moreover, the simple relationship (exponential) between PL intensity and pressure indicates that the relevant gas variable is closely related to the pressure. From the kinetic theory of gases we know that, for a given gas and temperature, several microscopic quantities are proportional to the macroscopic pressure: the density of gas molecules, the number of collisions against the walls, and the energy transmitted by these collisions. If the effect of the gas were linked to one of these

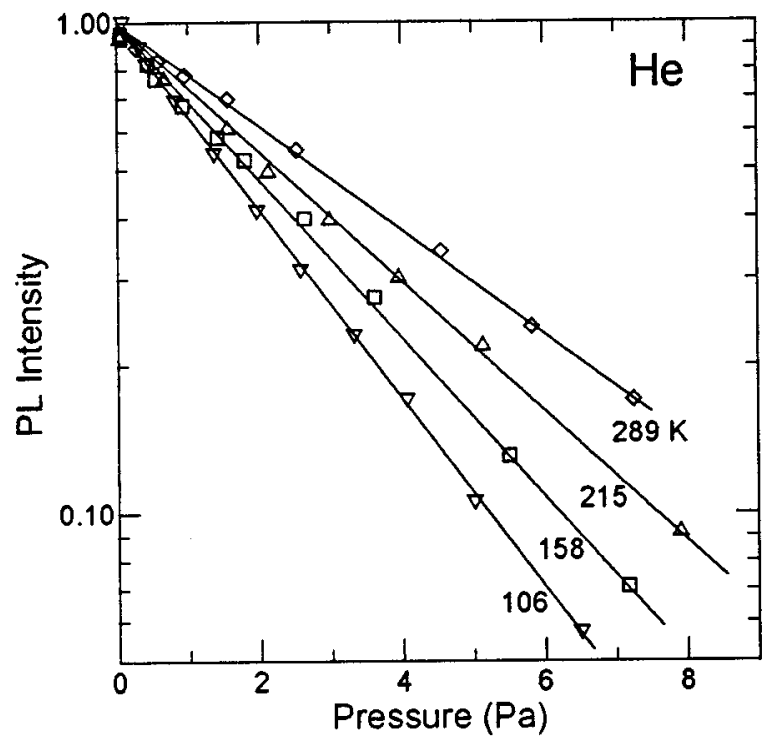

FIG. 3. Quenching of the PL intensity at different temperatures. 


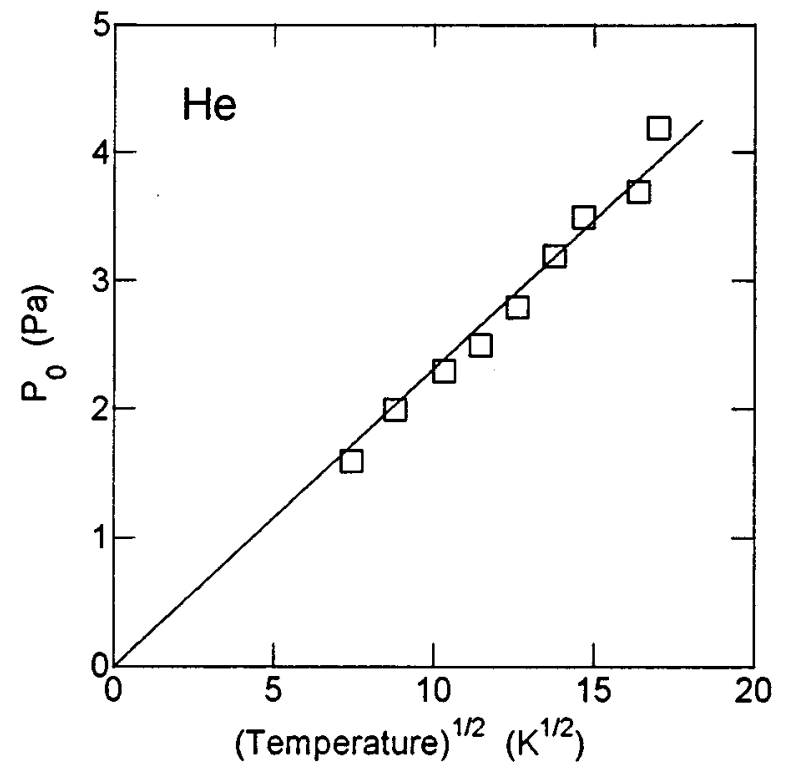

FIG. 4. The evolution of $P_{0}$ with the temperature of the surrounding gas. The proportionality to the square root of temperature indicates that the quenching is proportional to the number of gas collisions independent of their energy.

quantities, then the characteristic pressure would depend on the molecular mass, $m$, and temperature, $T$, following the general relationship

$$
P_{0} \propto T^{j} m^{l},
$$

where the exponents $j$ and $l$ would reveal the microscopic quantity involved.

Let us suppose that the quenching of the luminescence depends on the number of collisions per unit time and unit area, $\mathbf{N}$, irrespective of the gas, the momentum, and the energy. Under this hypothesis, we can write

$$
I_{\mathrm{PL}}=I_{0} e^{-N / N_{0}},
$$

where the constant $\mathbf{N}_{0}$ is independent of the gas and temperature. For an ideal gas, ${ }^{16}$

$$
P=N \sqrt{2 \pi} \sqrt{k T \mathbf{m}},
$$

where $k$ is Boltzman's constant. Substituting Eq. (5) into Eq. (2) and comparing it with Eq. (4) we can see that

$$
P_{0} \propto T^{1 / 2} \mathbf{m}^{1 / 2} \text {. }
$$

Therefore, for the case of collisions, $j$ and $l$ in Eq. (3) are equal to $1 / 2$. The exponents for the other microscopic quantities can be obtained similarly. For the density of gas molecules $j=1$ and $l=0$, whereas for the energy that the collisions can deliver $j=-1 / 2$ and $l=1 / 2$. So, from the dependence of $P_{0}$ on $T$ and $m$ the relevant microscopic quantity can be elucidated.

\section{B. Comparison with experiment}

In Fig. 4 we have summarized the dependence of $P_{0}$ at different temperatures for $\mathrm{He}$ by plotting $P_{0}$ vs $T^{1 / 2}$. This axis was chosen to test the hypothesis involving the number

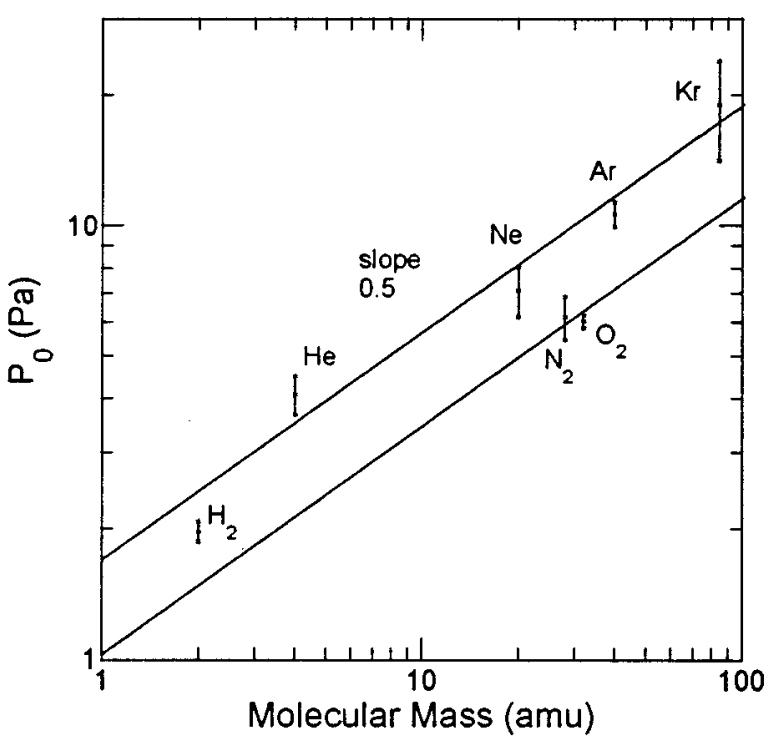

FIG. 5. The evolution of $P_{0}$ with the molecular mass of the gas. The proportionality to the square root of mass indicates that the quenching is proportional to the number of gas collisions independent of their momentum.

of collisions. The high correlation of the experimental points with a proportional relationship indicates that this hypothesis is valid. As temperature is a measure of the kinetic energy of the gas molecules, it follows that the PL quenching is independent of the energy of the molecules before the collision.

In Fig. 5 a $\log -\log$ plot summarizes the dependency of $P_{0}$ on the molecular mass reported in Fig. 2. The monoatomic and diatomic gases show a reasonable correlation with $m^{1 / 2}$. The small discrepancies can be ascribed to unknown refinements not considered in the hypothesis of collisions. Within each class of gases (mono- or diatomic) the energy before the collision is the same $(300 \mathrm{~K})$. The collision parameter that is gas dependent is its linear momentum. Figure 5 shows that the PL quenching is independent of the momentum before the collision. On the other hand, the smaller values of $P_{0}$ for the diatomic gases indicate that their collisions are more efficient. For an equivalent molecular mass, the characteristic pressure of a monoatomic gas is, roughly, $5 / 3$ that of the diatomic. On the other hand, from the equipartition principle, at any temperature, the energy of the diatomic gases is $5 / 3$ that of the monoatomic. Apparently their different behavior is attributable to the energy per molecule. However, as deduced from Fig. 4, there is no dependence on the energy before the collision. So, we conclude that the efficiency depends on the energy the gas molecules receive from the sample (after the collision).

In conclusion, the formula that best describes the PL quenching is not Eq. (2) but, rather, Eq. (4), because the "characteristic number of collisions," $N_{0}$, turns out to be a parameter characteristic of the material, independent of the gas and the temperature. In the usual experimental conditions (laser intensity of about $5-10 \mathrm{~mW} / \mathrm{mm}^{2}$ ) $N_{0}$ can change by a factor of 3 from sample to sample, and we can write

$$
N_{0} \approx \xi 1 \times 10^{23} \mathrm{~m}^{-2} \mathrm{~s}^{-1},
$$


where $\xi=2 / 3$ and $2 / 5$ for the mono- and diatomic gases, respectively.

\section{SUMMARY AND PERSPECTIVES}

The pressure quenching of the PL emitted by Si nanopowder grown by PECVD was analyzed. This effect was measured for various gases and at different temperatures from 60 to $300 \mathrm{~K}$. In all cases, the characteristic pressure $P_{0}$ [Eq. (2)] depends on the gas and on the temperature. A uniform behavior to explain these results is obtained if the number of gas collisions is considered. It was demonstrated that the quenching of the PL due to a given number of collisions is independent of their momentum and energy, and can be described with Eq. (4). The greater efficiency of the diatomic molecules can be ascribed, apparently, to the greater energy they receive from the sample after the collision. At this point it is tempting to assign the PL quenching simply as an effect related to the local temperature of the sample. The presence of the gas would reduce the local heating and this would reduce the PL intensity. At present we have no direct proof of this hypothesis so we have left the resolution of this to a future article.

Although no more is known about the effect of gas collisions, it must be of a very different nature than the effect of the gas on porous silicon. In porous silicon it seems that the PL quenching is explained by the adsorption of polar molecules, ${ }^{11,12}$ whereas the effect of nonpolar gases is much less pronounced. ${ }^{11}$ Furthermore, the pressures needed for PL quenching are much higher in porous silicon (at least 10 mbar) than in Si nanopowder.

\section{ACKNOWLEDGMENT}

This work was supported by the Spanish Programa Nacional de Materiales under CICYT Project Nos. MAT1511/ 94-CE, MAT95-2024-E, and MAT96-1194-C02.

${ }^{1}$ L. T. Canham, Appl. Phys. Lett. 57, 1046 (1990).

${ }^{2}$ K.-H. Li, C. Tsai, S. Shih, T. Hsu, D. L. Kwong, and J. C. Campbell, J. Appl. Phys. 72, 3816 (1992).

${ }^{3}$ S.-G. Lee, B.-H. Cheong, K.-H. Lee, and K. Y. Kang, Phys. Rev. B 51, 1762 (1995).

${ }^{4}$ M. S. Brandt, H. D. Fuchs, M. Stutzmann, J. Weber, and M. Cardona, Solid State Commun. 81, 307 (1992).

${ }^{5}$ C. Tsai, K.-H. Li, J. Srathy, S. Shih, J. C. Campbell, B. K. Hance, and J. M. White, Appl. Phys. Lett. 59, 2814 (1992).

${ }^{6}$ H. Mosisaki, H. Hashimoto, F. W. Ping, H. Nozawa, and H. Ono, J. Appl. Phys. 74, 2977 (1993).

${ }^{7}$ S. Furukawa and T. Miyasato, Phys. Rev. B 36, 5726 (1988).

${ }^{8}$ C. Courteille, J.-L. Dorier, J. Dutta, Ch. Hollenstein, A. A. Howling, and T. Stoto, J. Appl. Phys. 78, 61 (1995).

${ }^{9}$ J. Costa, P. Roura, G. Sardin, J. R. Morante, and E. Bertran, Appl. Phys. Lett. 64, 463 (1994)

${ }^{10}$ P. Roura, J. Costa, G. Sardin, J. R. Morante, and E. Bertran, Phys. Rev. B 50, 18124 (1994).

${ }^{11}$ N. Kuroda, Y. Matsuda, S. Nakajima, I. Taketsu, and N. Ookubo, J. Appl. Phys. 78, 3520 (1995).

${ }^{12}$ M. Ben-Chorin and A. Kux, Appl. Phys. Lett. 64, 481 (1994).

${ }^{13}$ J. Zeman, M. Zigone, G. L. J. A. Rikken, and G. Martinez, Solid State Commun. 96, 503 (1995).

${ }^{14}$ J. Costa, P. Roura, N. A. Sulimov, G. Sardin, J. Campmany, J. R. Morante, and E. Bertran, Mater. Sci. Technol. 11, 707 (1995).

${ }^{15}$ P. Roura, J. Costa, N. A. Sulimov, J. R. Morante, and E. Bertran, Appl. Phys. Lett. 67, 2830 (1995).

${ }^{16}$ A. Arjaron, I. Marferina, and E. Mikulin, Sistemas Criogénicos (Mir, Moscow, 1988).

${ }^{17}$ J. Costa, Ph.D. thesis, Universitat de Barcelona, 1995.

${ }^{18}$ J. Dutta, W. Bacsa, and Ch. Hollenstein, J. Appl. Phys. 77, 3729 (1995). 